\title{
Prevalence and genetic diversity of astroviruses in children with and without diarrhea in São Luís, Maranhão, Brazil
}

\author{
Yvone B Gabbay/ ${ }^{+}$, Cláudia Regina NE da Luz*, Ivete V Costa, \\ Elielma L Cavalcante-Pepino, Maisa S Sousa, Krynssya K O liveira, \\ Ana Lucia Monteiro Wanzeller, Joana D'Arc P Mascarenhas, José Paulo G Leite**, \\ Alexandre C Linhares
}

\begin{abstract}
Seção de Virologia, Instituto Evandro Chagas, Secretaria de Vigilância em Saúde, Ministério da Saúde, BR 316 Km 07, s/nº 67030000 Ananindeua, PA, Brasil *Hospital Universitário Materno Infantil, São Luís, MA, Brasil **Laboratório de Virologia

Comparada, Departamento de Virologia, Instituto Oswaldo Cruz-Fiocruz, Rio de Janeiro, RJ, Brasil
\end{abstract}

Human astroviruses (HAstV) have been increasingly identified as important etiological agents of acute gastroenteritis in children up to five years old. The aim of this study was to determine the prevalence and genotype diversity of HAstV in children with symptomatic and asymptomatic infections in São Luís, Maranhão, Brazil. From June 1997 to July 1999 a total of 183 fecal samples 84 from symptomatic and 99 from asymptomatic children were tested by enzyme immunoassay for HAstV. Prevalence rates were found to be 11 and $3 \%$ for symptomatic and asymptomatic children, respectively. Reverse transcription-polymerase chain reaction (RT-PCR) was carried out in 46 specimens (26 symptomatic and 20 asymptomatic) including the 12 samples that were positive by enzyme immunoassay (EIA). The overall positivity yielded by both methods was 8\% (15/184); of these, 11\% (9/84) for symptomatic and 5\% (5/99) for those without symptoms or signs. Sequence analysis of amplicons revealed that HAstV-1 genotype was the most prevalent, accounting for $60 \%$ of isolates. Genotypes 2, 3, 4, and 5 were also detected, as one single isolate (10\%) for each type. Variations in the sequences were observed when Brazilian isolates were compared to prototype strains identified in the United Kingdom. No seasonal pattern of occurrence was observed during these two years of study, and peak detection rate was observed in children aged between 3 and 6 months in the symptomatic group, and between 18 and 24 months in the controls.

Key words: human astroviruses (HAstV) - diarrhea - children - São Luís, Maranhão - Brazil

Human astroviruses (HAstVs) are members of the Astroviridae family which are recognized as one of the most common cause of viral gastroenteritis in infants and young children (Glass et al. 1996).

HAstV derive their name from a morphologically characteristic five- or- six point star shape they display when visualized by electron microscopy (EM) (Appleton \& Higgins 1975, Madeley 1995). The HAstV virion is composed of a single non-enveloped capsid layer of 27 to 34 $\mathrm{nm}$ in diameter (Matsui \& Greenberg 2001). The genome consists of a single-stranded (ss), positive sense, polyadenylated RNA with 6.8-7.8 kilobases in length. Three open reading frames (ORFs) have been identified: ORF1a and ORF1b, which encode viral protease and polymerase, respectively; and ORF2, which encodes the capsid protein precursor (Willcocks et al. 1994, Jiang et al. 1993).

HAstVs are classified into eight genotypes based on the nucleotide sequence of a 348-bp region of ORF2 that have shown a good correlation with the eight established serotypes (Appleton \& Higgins 1975, Noel et al. 1995, Matsui \& Greenberg 2001). Typing surveys have shown

Financial support: IEC/SVS, IOC-Fiocruz, Funtec-PA, Pibic/ $\mathrm{CNPq}$

${ }^{+}$Corresponding author. E-mail: yvonegabbay@iec.pa.gov.br Received 2 June 2005

Accepted 26 October 2005 that type 1 is the most prevalent, followed by types 2, 3, 4, and 5 (Lee \& Kurtz 1994, Glass et al. 1996, Mustafa et al. 2000, Sakamoto et al. 2000).

Usually HAstVs infect young children, however, outbreaks of diarrhea involving elderly people, military recruits and immunocompromised patients have been reported (Grohmann 1993, Glass et al. 1996, Belliot et al. 1997). The main symptom of infection caused by HAstV is watery diarrhea, which is often associated with vomiting, fever, anorexia, and abdominal pain (Matsui \& Greenberg 2001).

Reports on HAstV infection have already been described in different cities of Brazil, showing that HAstV prevalence rates range between 2.8 and $33 \%$. These studies focused on surveillance for gastroenteritis in hospitals and day-care centers, as well as on self-limited outbreaks (Leite et al. 1991, Timenetsky et al. 1993, Tanaka et al. 1994, Silva et al. 2001, Cardoso et al. 2002).

The main purpose of this report is to provide epidemiological and clinical data on the occurrence of HAstV infection among diarrheic and non-diarrheic children aged up to two years, living in São Luís, Maranhão, Northeast region of Brazil. We have also analyzed the partial nucleotide sequences of ORF2 region to determine the prevalence of HAstV genotypes.

\section{MATERIALS AND METHODS}

Study subjects - From June 1997 to July 1999 a hospital-based surveillance was conducted at the Infantile 
Maternal University Hospital from Federal University of Maranhão (HUMI/UFMA), including children under two years of age, both sexes, who lived under poor sanitation conditions in the central area of São Luís, Maranhão, a city located in the Northeast region of Brazil.

Stool specimens - At enrollment an informed consent was obtained from either parents or guardians of participating children, and a complete standardized questionnaire designed to gathering main clinical and epidemiological data was completed by a pediatrician. Diarrheic children (symptomatic) were defined as those subjects who developed diarrhea, defined as the passage of $\geq 3$ looser than normal stools in a $24 \mathrm{~h}$ period, lasted no longer than 14 days, and coursed with dehydration. A group of children who attended an outpatient pediatric clinic of HUMI/UFMA, without diarrhea, were enrolled as controls (asymptomatic). Of 245 fecal specimens obtained during the surveillance study, 183 samples had a sufficient quantity to be tested for HAstV 84 from children with diarrhea and 99 from those without diarrhea. These samples were stored at $-20^{\circ} \mathrm{C}$ until they were sent to the Instituto Evandro Chagas, Belém, Pará, where they were processed.

Enzyme immunoassay (EIA) - All 183 samples were screened for the presence of HAstV antigen by a commercial HAstV EIA kit (IDEIA ${ }^{\mathrm{TM}}$, DakoCytomation, Ely, UK). These samples were also tested for the presence of rotavirus (RV) and adenovirus (AdV) by commercially available EIAs (Premier-Rotaclone ${ }^{\mathrm{TM}}$ and Adenoclone ${ }^{\mathrm{TM}}$, Meridian Bioscience Inc., US).

Electron microscopy (EM) - Briefly, a 10\% fecal suspension was made in water and clarified by low-speed centrifugation $(1500 \times \mathrm{g}$ for $15 \mathrm{~min})$. The supernatants were collected and centrifuged at $14,000 \times \mathrm{g}$ for $40 \mathrm{~min}$. The resulting pellets were resuspended in $50 \mu \mathrm{l}$ of water and $10 \mu \mathrm{l}$ were placed on a carbon-formvar-coated copper grid. Staining was done with potassium phosphotungstic acid pH 7.0. Samples were examined under a Zeiss EM 900 transmission electron microscope.

RNA extraction and amplification assays - Viral ssRNA was extracted from $200 \mu \mathrm{l}$ of a $10 \%$ fecal suspension using silica/guanidine thiocyanate, as described by Boom et al. (1990), including modifications introduced by Cardoso et al. (2002).

RT-PCR for HAstV was carried out in 44 samples using primers (Mon 269 and Mon 270) directed towards the ORF2 region, and the amplification conditions were those as described by Noel et al. (1995), except for the RT reaction where a random initiator [hexamer pd $(\mathrm{N})_{6}-50 \mathrm{~A}_{260}$ units - Amersham Biosciences] was utilized to obtain the cDNA product. RT-PCR products were resolved on a $1 \%$ agarose gel, followed of ethidium bromide staining and photo-documentation was done in a Gel Doc 1000 (BioRad). Samples showing a specific amplicon of $449 \mathrm{bp}$ were considered as positive.

Water was used as a negative control in all procedures and recommended manipulations for PCR techniques were strictly followed as a precaution to avoid false-positive results. A HAstV-1 strain, kindly donated by Dr Roger Glass, CDC, Atlanta, was used as a positive control.

Sequencing of RT-PCR astrovirus amplicons - The amplicons were purified with Concert ${ }^{\mathrm{TM}}$ Rapid PCR Purification System (Invitrogen), in accordance with the manufacturer's instructions. The nucleotide sequence was determined by direct cycle sequencing using the Big Dye Terminator Cycle Sequencing Ready Reaction Kit (Applied Biosystems) and the primers Mon 269 and Mon 270. The purification of the amplicon was done by isopropanol/ ethanol $(75 / 70 \%)$ precipitation. The products were analyzed on an automatic ABI Prism 3100 DNA Sequencer (Applied Biosystems). Sequence data from both strands were aligned and edited using the BioEdit Sequence Alignment Editor (v. 6.0) program and compared with eight prototype sequences: HAstV1 (L23513), HAstV2 (L13745), HAstV3 (L38505), HAstV4 (L38506), HAstV5 (U15136), HAstV6 (L38507), and HAstV7 (L38508), Oxford reference strains and HAstV-8 (Z66541), United Kingdom reference, obtained from GenBank at the National Center for Biotechnology Information, US (www.ncbi.nlm.nih.gov), using the BLAST program. The dendrogram was constructed by neighbor-joining method using the Mega (v. 3.0) software, supported by bootstrap using 1000 replicates.

Nucleotide sequence accession numbers - The nucleotide sequences determined in this study have been deposited in the GenBank database and assigned the accession numbers from DQ071648 to DQ071656.

\section{RESULTS}

From a total of 183 samples analyzed by the EIA, 9/84 (11\%) from diarrheic children and 3/99 (3\%) from nondiarrheic children were positive for HAstV $(p=0.072)$. RTPCR was carried out in 46 specimens (12 which reacted HAstV-positive by EIA and 34 showing an optical density value of at least twice that of the negative control). Taking both methodologies together (EIA and RT-PCR), $14(8 \%)$ out of the 183 samples [ $9 / 84(11 \%)$ from diarrheic and 5/99 (5\%) from non-diarrheic] were positive for HAstVs (Table I). Four samples were positive by EIA only, after being tested at least twice. In addition, two of them were confirmed as positive by EM, where particles with typical morphology of HAstV were visualized.

\section{TABLE I}

Detection of human astrovirus by enzyme immunoassay (EIA) and reverse transcription followed by polymerase chain reaction (RT-PCR) in fecal specimens from 183 children, São Luís, Maranhão, September 1997 to July 1999

\begin{tabular}{lcclcc}
\hline & \multicolumn{2}{c}{ Diarrheic $(84)^{a}$} & & \multicolumn{2}{c}{ Non-diarrheic (99) ${ }^{a}$} \\
\cline { 2 - 3 } \cline { 5 - 6 } EIA & \multicolumn{2}{c}{ RT-PCR (\%) } & & \multicolumn{2}{c}{ RT-PCR $(\%)$} \\
\cline { 2 - 3 } \cline { 5 - 6 } Positive & $7(8)$ & $2(3)$ & & $1(1)$ & $2(2)$ \\
Negative & - & $75(89)$ & & $2(2)$ & $94(95)$ \\
\hline
\end{tabular}

$a$ : number of fecal specimens tested. 
Mixed infection was observed in five diarrheic HAstVpositive children: three of whom were also excreting RV, one was enteric AdV-positive, and a third patient was found to be also positive for both RV and non-enteric $\mathrm{AdV}$. A mixed infection involving $\mathrm{HAstV}$ and $\mathrm{RV}$ was also identified in a non-diarrheic child (data not shown).

Nucleotide sequence data were obtained from nine out of ten RT-PCR-positive samples. All HAstV-1 strains were gathered in the period of March-July 1999. A phylogenetic tree from analysis of a 348 bp region of the capsid is shown in Fig. 1. It can be seen that all HAstV-1 strains were clustered into one major lineage containing a number of minor branches. Four HAstV strains were clustered with HAstV-2, 3, 4, and 5 strains. All major branches demonstrated a $100 \%$ bootstrap confidence level. According to the segregation of the nucleotide and deduced amino acid sequences, the five HAstV genotypes demonstrated divergences that ranged from 22.1 to $28.8 \%$ and from 3.1 to $10 \%$, respectively (data not shown).

Variation in the nucleotide sequence, but not in the amino acid sequence, was more evident between the São Luís isolates and the prototype strains used in the phylogenetic analysis (Fig. 1). In general, limited nucleotide variation was observed in the São Luís genotype 1 isolates, which displayed a maximum of $0.9 \%$ nucleotide sequence diversity. The variation between these samples and the prototype HAstV-1 reached a maximum of $2.4 \%$. All the nucleotide sequence changes within the São Luís
HAstV-1 strains were silent with respect to amino acid coding, except for one specimen (MA167) (data not shown). This sample contained a Lys $\rightarrow$ Arg change at amino acid 56 of the capsid protein ( $0.9 \%$ of diversity). São Luís HAstV-2, 3, 4, and 5 strains displayed a variation in their nucleotide sequence, in comparison with their prototypes, of $2.3,2.4,6.3$, and $4.4 \%$, respectively. In considering the amino acid sequence, the variations found among the four types, in comparison with their corresponding prototypes were $0.0,0.9,0.9$, and $1.7 \%$, respectively.

Seasonal pattern of occurrence was not observed over the two years of survey, with positive cases being detected in the months of September-December 1997, October 1998, and March-June 1999 (Fig. 2A). In the non-diarrheic children HAstV was found in October 1997, May and November 1998, and June and July 1999, with one positive sample per month (Fig. 2B).

The age distribution of HAstV infection is shown in Table II. The peak detection rate was observed in $18 \%$ of the symptomatic children aged 0 to 6 months old, and 18 to 24 months old (25\%) for the non-diarrheic patients. The main symptoms observed in the nine HAstV-positive diarrheic patients were fever and vomiting (89\%) (Table III).

\section{DISCUSSION}

This study describes the prevalence and genotype distribution of HAstV infections in sporadic cases of in-

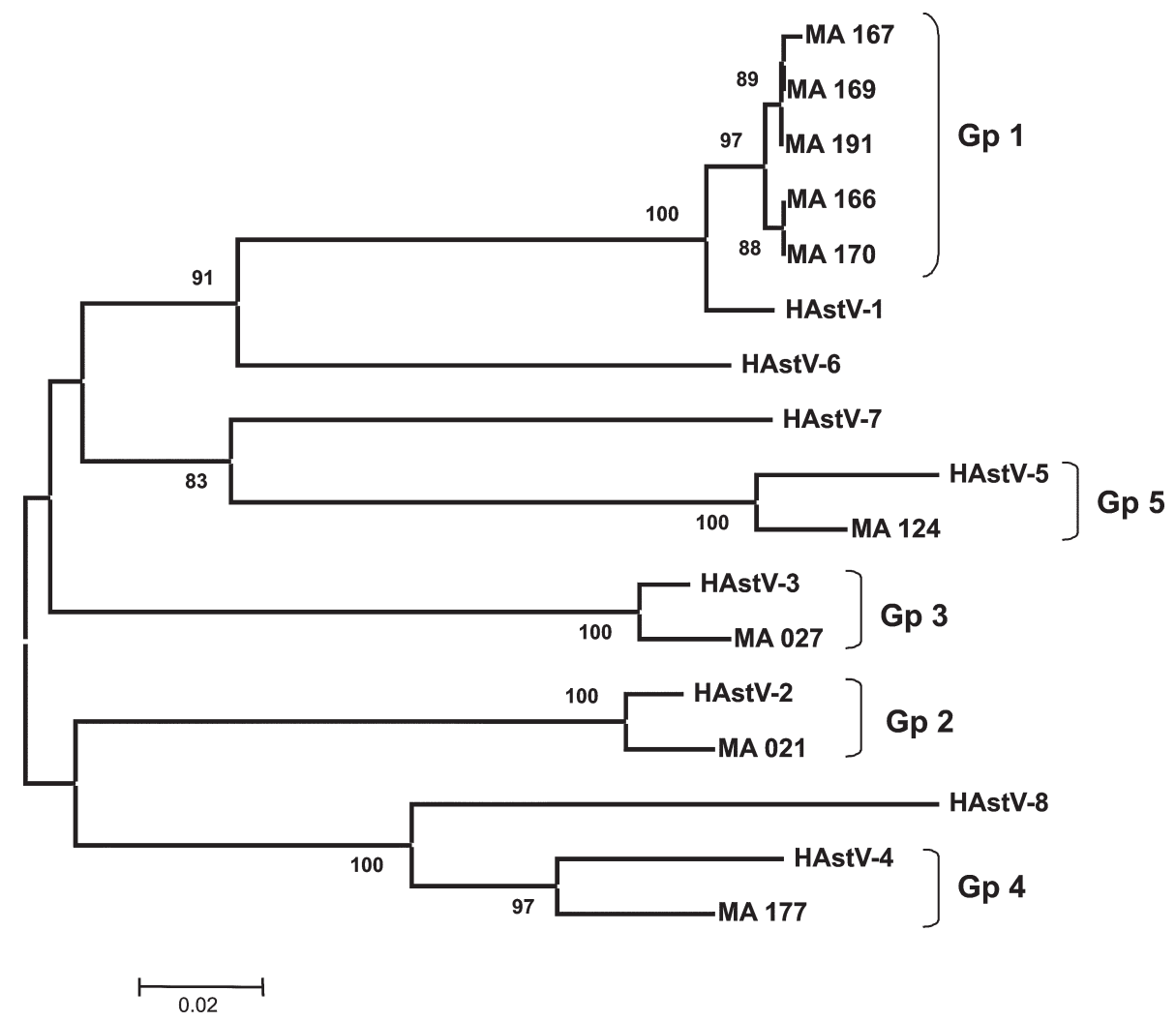

Fig. 1: phylogenetic tree of a 348-bp ORF2 region of human astrovirus (HAstV) strains from São Luís, Maranhão, from June 1997 to July 1999 and standard HAstV strains (HAstV-1 to HAstV-8) isolated in the United Kingdom. The scale bar represents a genetic distance of $2 \%$. The sequences were obtained from the GenBank database with the accession numbers listed in Materials and Methods. 


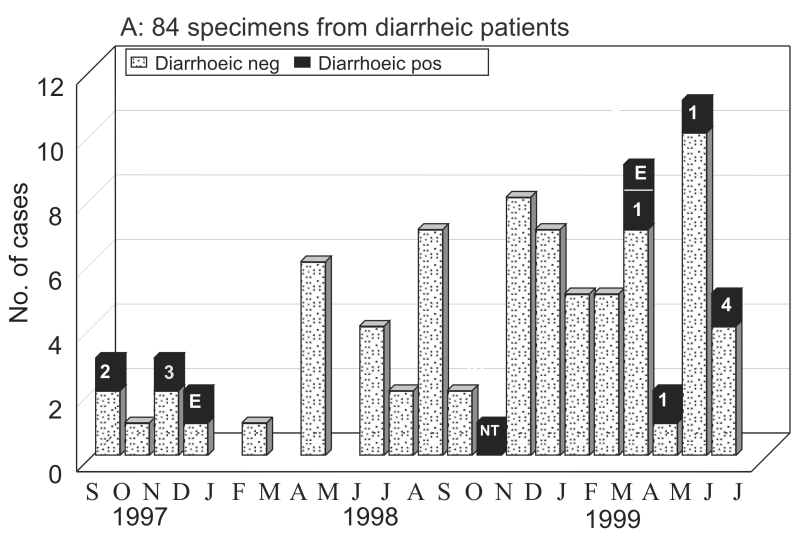

B: 99 specimens from non-diarrheic patients

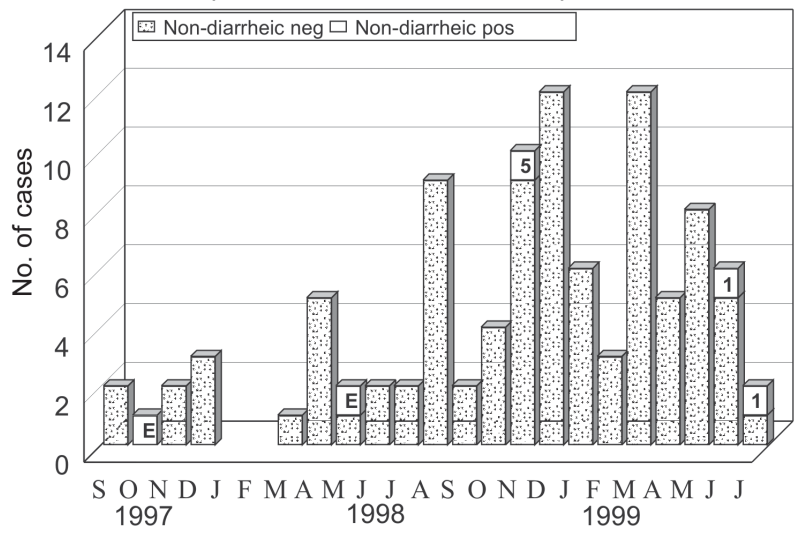

Fig. 2: temporal distribution and human astrovirus (HAstV) genotypes detected in faecal specimens from 183 children, São Luís, Maranhão, from September 1997 to July 1999. 1, 2, 3, 4, and 5: types of astrovirus detected during the study; E: ELISA positive and PCR negative; NT: not typed

TABLE II

Frequency of human astrovirus (HAstV), according to age, in children from São Luís, Maranhão, June 1997 to July 1999

\begin{tabular}{lcc}
\hline & Diarrheic & Non-diarrheic \\
\cline { 2 - 3 } Age (months) & HAstV/Total $(\%)$ & HAstV/Total $(\%)$ \\
\hline $0-6$ & $2 / 11(18)$ & $1 / 12(8)$ \\
$>6-12$ & $4 / 48(8)$ & $2 / 56(4)$ \\
$>12-18$ & $2 / 18(11)$ & $0 / 23$ \\
$>18-24$ & $1 / 7(14)$ & $2 / 8(25)$ \\
\hline Total & $9 / 84(11)$ & $5 / 99(5)$ \\
\hline
\end{tabular}

fantile gastroenteritis in São Luís, Maranhão, Brazil, from September 1997 to July 1999. The HAstV prevalence rate found among the diarrheic children (11\%) was similar to that observed in Madrid, Spain (Dalton et al. 2002), but lower than that for Chilean children (16\%) (Gaggero et al. 1998). Our data showed rates higher than those reported previously (4-5\%) for other countries; for instance, Melbourne, Central Australia, Barcelona, Mexico, and Bangladesh (Polombo et al. 1996, Unicomb et al. 1998, Guix et al. 2002, Schnagl et al. 2002, Mendez-Toss et al.
TABLE III

Clinical symptoms observed in diarrheic children with human astrovirus (HAstV) infection, São Luís, Maranhão, June 1997 to July 1999

\begin{tabular}{lc}
\hline Symptoms & $\begin{array}{c}\text { HAstV positive } \\
(\mathrm{n}=9)(\%)\end{array}$ \\
\hline Vomiting & $8(89)$ \\
Fever & $8(89)$ \\
Anorexia & $7(78)$ \\
Abdominal pain & $6(67)$ \\
Mild dehydration & $4(44)$ \\
Moderate dehydration & $5(56)$ \\
Severe dehydration & -
\end{tabular}

$\bar{a}$ : mixed infection was observed in five cases: three with rotavirus $(\mathrm{RV})$ one with enteric adenovirus (AdV) and one with both RV and AdV.

2004). With regards to the prevalence of HAstV infection in Brazil, studies conducted in a public hospital in Goiânia, Goiás (Cardoso et al. 2002), São Paulo (Timenetsky et al. 1993), and Rio de Janeiro (Leite et al. 1991) yielded prevalence rates of 3-5\%, which were lower than those reported for São Luís. The prevalence of HAstV infection among non-diarrheic children (5\%) was similar to those previously reported in various countries such as Mexico (3\%), Bangladesh (2\%), South Africa (2\%), Guatemala (2\%), and Thailand (2\%) (Herrmann et al. 1991, Cruz et al. 1992, Steele et al. 1998, Unicomb et al. 1998, Méndez-Toss et al. 2004).

Of note, co-infection involving HAstV and RV were detected in $36 \%$ of our samples, a rate which is greater than that of $17 \%$ observed in Barcelona, Spain, and Providence, US (Dennehy et al. 2001, Guix et al. 2002). The high rate of mixed infection was probably due to the poor sanitation conditions which prevail in the environments where these children are living. Dual infection involving HAstV and RV was also reported recently among Mexican children, suggesting this as being a relatively common finding (Méndez-Toss et al. 2004). In the Mexican study, $\mathrm{HAst} \mathrm{V}$ was found at similar frequencies in both RV-positive and RV-negative diarrheic children, suggesting that no synergy occurs among infections caused by these two viruses (Méndez-Toss et al. 2004).

In this study, HAstVs were detected in 14 fecal specimens, four $(28.6 \%)$ of which positive by EIA only, two (14.3\%) by RT-PCR only, and eight (57.1\%) reacting positive by both techniques. The differences in detection rates between these two techniques may be explained by the fact that EIA indicates the presence of both soluble and particle-associated HAstV antigen, while RT-PCR assays detect nucleic acid in intact virus particles. A few hypotheses should be considered in explaining such divergent results: (a) a possible false positive result; (b) nucleotide sequence differences in the primer hybridization region; and (c) the presence of inhibitors in the RT-PCR. This was previously observed by Maldonado et al. (1998) and Silva et al. (2001). It is worth mentioning that the four samples positive by EIA only were also examined by EM and virus particles with morphology typical of HAstV could be visualized in two of them. It seems likely that the presence 
of defective viral particles at concentrations lower than that of EM sensitivity may have accounted for the negative results from examining two other samples (Silva et al. 2001).

As reported elsewhere (Polombo et al. 1996, Gaggero et al. 1998, Mustafa et al. 2000, Silva et al. 2001, Cardoso et al. 2002, Dalton et al. 2002, Guix et al. 2002, Espul et al. 2004), HAstV-1 was found to be the most prevalent genotype, with rates ranging from 38 to $100 \%$.

It is worth mentioning that other HAstV genotypes (2, 3,4 , and 5) were circulating concurrently with the predominant genotype 1 . The genotypic complexity of cocirculating strains differs from the findings of Noel et al. (1995) who demonstrated a clustering of strains with the same sequence circulating in various settings worldwide.

Genetic diversity was evident when we compared nucleotide and amino acid sequences of samples isolated in São Luís to the prototype strains from the United Kingdom. The detection of five different types co-circulating during a two-year period suggests that the genotype distribution of HAstV in São Luís varies throughout the time. This may have implications for the future development of possible HAstV vaccines.

The seasonal pattern of HAstV infection was well documented in studies carried out in both temperate and tropical regions of the world, where this virus was found to prevail either throughout the winter months or during the rainy season (Matsui \& Greenberg 2001). In our study, which was conducted in a tropical region, we observed that most of the HAstV infections clustered in two periods: September to December 1997 and March to July 1999. The first period corresponds to a relatively dry season, with a temperature variation in the range of 27.5 to $28^{\circ} \mathrm{C}$; and the second period, a typical rainy season, with a heavy rainfall $(200$ to $600 \mathrm{~nm})$ and a temperature of around $27^{\circ} \mathrm{C}$. It is noteworthy that all HAstV-1 strains were found during March-July 1999.

In this study, the highest infection rate (18\%) among diarrheic children was observed in the 0 to 6 months age group. These results agree with those of Giordano et al. (2004) in Cordoba, Argentina, who reported a 24\% HAstVpositivity in children 4 to 6 months old. Conversely, our results differ from those obtained in a 3-year study conducted in Barcelona, Spain (Guix et al. 2002), where the peak incidence was observed in older children, aged between 2 and 4 years, yielding a $9 \%$ positivity rate. In the non-diarrheic group HAstV was detected more frequently in older children, aged 18 to 24 months (25\%). It should be pointed out, however, that such a peak incidence may have occurred by chance, owing to the low numbers in each group.

In our study, the percentages of vomiting $(88.9 \%)$, fever $(88.9 \%)$, and moderate dehydration $(56 \%)$ were higher than those reported in a study in Madrid, Spain $(65,39$, and $24 \%$, respectively) conducted at an emergency room (Dalton et al. 2002).

Further surveillance studies to fully assess the epidemiological importance of HAstV among diarrheic and nondiarrheic children in São Luís are worth to be done, including a larger sample size. Since a RV vaccine is expected to become available soon, the search for other potential enteropathogens such as HAstVs and noroviruses will gain increasing epidemiological importance.

\section{ACKNOWLEDGMENTS}

To technical assistance provided by Antonia S Alves and Antônio de Moura. To support given by Dr Olinda Macedo, Dr Darleise de Souza Oliveira, and Dr Maria de Lourdes C Gomes for DNA sequencing.

\section{REFERENCES}

Appleton H, Higgins PG 1975. Viruses and gastroenteritis in infants. Lancet 1: 1297.

Belliot G, Laveran H, Monroe SS 1997. Outbreak of gastroenteritis in military recruits associated with serotype 3 astrovirus infection. J Med Virol 51: 101-106.

Boom R, Sol CJA, Salimans MMM, Jansen CL, Wertheim-Van Dillen PME, Van Der Noordaa J 1990. Rapid and simple method for purification of nucleic acids. J Clin Microbiol 28: 495-503.

Cardoso DDP, Fiaccadori FS, Souza MBLD, Martins RMB, Leite JPG 2002. Detection and genotyping of astroviruses from children with acute gastroenteritis from Goiânia, Goiás, Brazil. Med Sci Monit 8: CR624-628.

Cruz JR, Bartlett AV, Herrmann JE, Caceres P, Blacklow NR, Cano F 1992. Astrovirus-associated diarrhea among Guatemalan ambulatory rural children. J Clin Microbiol 30: 11401144.

Dalton RM, Roman ER, Negredo AA, Wilhelmi ID, Glass RI, Sànchez-Fauquier A 2002. Astrovirus acute gastroenteritis among children in Madrid, Spain. Pediatr Infect Dis J 21: 1038-1041.

Dennehy PH, Nelson SM, Spangenberger S, Noel JS, Monroe SS, Glass RI 2001. A prospective case-control study of the role of astrovirus in acute diarrhea among hospitalized young children. J Infect Dis 184: 10-15.

Espul A, Martinez N, Noel JS, Cuello H, Abrile C, Grucci S, Glass R, Berke T, Matson DO 2004. Prevalence and characterization of astroviruses in Argentinean children with acute gastroenteritis. J Med Virol 72: 75-82.

Gaggero A, O'Ryan M, Noel JS, Glass RI, Monroe SS, Mamani N, Prado V, Avendano LF 1998. Prevalence of astrovirus infection among Chilean children with acute gastroenteritis. J Clin Microbiol 36: 3691-3693.

Giordano MO, Martinez LC, Isa MB, Paez Rearte M, Nates SV 2004. Childhood astrovirus-associated diarrhea in the ambulatory setting in a public hospital in Cordoba city, Argentina. Rev Inst Med Trop São Paulo 46: 93-96.

Glass RI, Noel JS, Mitchell, D, Herrmann JE, Blacklow NR, Pickering LK, Dennehy P, Ruiz-Palacios G, De Guerreiro ML, Monroe SS 1996. The changing epidemiology of astrovirus-associated gastroenteritis: a review. Arch Virol 12: 287-300.

Grohmann GS, Glass RI; Pereira HG, Monroe SS, Hightower AW, Wleber R, Bryan RT 1993. Enteric viruses and diarrhea in HIV-infected patients. N Engl J Med 329: 14-20.

Guix S, Caballero S, Villena C, Bartolome R, Latorre C, Rabella N, Simo M, Bosch A, Pinto M 2002. Molecular epidemiology of astrovirus infection in Barcelona, Spain. J Clin Microbiol 40: 133-139. 
Herrmann JE, Taylor DN, Echeverria P, Blacklow NR 1991. Astrovirus as a cause of gastroenteritis in children. N Eng $J$ Med 324: 1757-1760.

Jiang B, Monroe SS, Koonin EV, Stine SE, Glass RI 1993. RNA sequence of astrovirus: distinctive genomic organization and a putative retrovirus-like ribosomal frameshifting signal that directs the viral replicase synthesis. Proc Natl Acad Sci USA 90: 10539-10543.

Lee TW, Kurtz JB 1994. Prevalence of human astrovirus serotypes in the Oxford region 1976-1992, with evidence for two new serotypes. Epidemiol Infect 112: 187-193.

Leite JPG, Barth O.M, Schatzmayr HG 1991. Astrovirus in faeces of children with acute gastroenteritis in Rio de Janeiro, Brazil. Mem Inst Oswaldo Cruz 86: 489-490.

Madeley CR 1995. Comparison of the features of astrovirus and calicivirus seen in samples of feces by electron microscopy. J Infect Dis 139: 519-523.

Maldonado Y, Cantwell M, Old M, Hill D, Sanchez ML, Logan L, Millan-Velasco F, Valdespino JL, Sepulveda J, Matsui S 1998. Population-based prevalence of symptomatic and asymptomatic astrovirus infection in rural Mayan infants. J Infect Dis 178: 334-339.

Matsui SM, Greenberg HB 2001. Astroviruses. In DM Knipe, PM Howley (eds), Fields Virology, 4th ed., Lippincott, Williams and Wilkins, Philadelphia, p. 875-893.

Medina SM, Guitierrez MF, Liprandi F, Ludert JE 2000. Identification and type distribution of astroviruses among children with gastroenteritis in Colombia and Venezuela. J Clin Microbiol 38: 3481-3483.

Méndez-Toss M, Griffin DD, Calva J, Contreras JF, Puerto FI, Mota F, Guiscafre H, Cedillo R, Munoz O, Herrera I, Lopez S, Arias CF 2004. Prevalence and genetic diversity of human astroviruses in Mexican children with symptomatic and asymptomatic infections. J Clin Microbiol 42: 151157.

Mustafa H, Palombo EA, Bishop RF 2000. Epidemiology of astrovirus infection in young children hospitalized with acute gastroenteritis in Melbourne, Australia, over a period of four consecutive years, 1995 to 1998. J Clin Microbiol 38: 1058-1062.

Noel JS, Lee TW, Kurtz JB, Glass RI, Monroe SS 1995. Typ- ing of human astroviruses from clinical isolates by enzyme immunoassay and nucleotide sequencing. J Clin Microbiol 33: 797-801.

Polombo EA, Bishop RF 1996. Annual incidence, serotype distribution, and genetic diversity of astrovirus isolates from hospitalized children in Melbourne, Australia. J Clin Microbiol 34: 1750-1753.

Sakamoto T, Negidhi H, Wang QH, Akihara S, Kim B, Nishimura S, Kaneshi K, Nakaya S, Ueda Y, Sugita K, Motohiro T, Nishimura T, Ushijima H 2000. Molecular epidemiology of astroviruses in Japan from 1995 to 1998 by reverse transcription-polymerase chain reaction with serotype-specific primers (1 to 8). J Med Virol 61: 326-331.

Schnagl RD, Belfrage K, Farrington R, Hutchinson K, Lewis V, Erlich J, Morey F 2002. Incidence of human astrovirus in Central Australia (1995 to 1998) and comparison of deduced serotypes detected from 1981 to 1998. J Clin Microbiol 40: 4114-4120.

Silva AM, Leite EG, Assis RM, Majerowicz S, Leite JP 2001. An outbreak of gastroenteritis associated with astrovirus serotype 1 in a day care center, in Rio de Janeiro, Brazil. Mem Inst Oswaldo Cruz, 96: 1069-1073.

Steele AD, Basetse HR, Blacklow NR, Herrmann JE 1998. Astrovirus infection in South Africa: a pilot study. Ann Trop Paediatr 18: 315-319.

Tanaka H, Kisielius JJ, Ueda M, Glass RI, Joazeiro PP 1994. Intrafamilial outbreak of astrovirus gastroenteritis in São Paulo, Brazil. J Diarrhoeal Dis Res 12: 219- 221.

Timenetsky MCST, Kisielius JJ, Grisi SJFE, Escobar AMU, Ueda M, Tanaka H 1993. Rotavírus, adenovírus, astrovírus, calicivírus e "small round vírus particles" em fezes de crianças, com e sem diarréia aguda, no período de 1987 a 1988, na grande São Paulo. Rev Inst Med Trop São Paulo 35: 275-280.

Unicomb LE, Banu NN, Azim T, Islam A, Bardhan PK, Faruque ASG, Hall A, Moe CL, Noel JS, Monroe SS, Albert MJ, Glass RI 1998. Astrovirus infection in association with acute, perdidtent and nosocomial diarrhea in Bangladesh. Pediatr Infect Dis J 17: 611-614.

Willcocks MM, Brown TDK, Madeley CR, Carter MJ 1994. The complete sequence of a human astrovirus. J Gen Virol 75: 1785-1988. 